\title{
Comparison of Human Menopousal Gonadotropin with Recombinant Follicle Stimulating Hormone in Ovulation Stimulation During Intrauterine Insemination
}

\author{
Eddy Hartono \\ Department of Obstetrics and Gynecology, Faculty of Medicine, Hasanuddin University, Makassar, South Sulawesi, Indonesia
}

\section{Email address:}

eddyhartono_spog@yahoo.com

\section{To cite this article:}

Eddy Hartono. Comparison of Human Menopousal Gonadotropin with Recombinant Follicle Stimulating Hormone in Ovulation Stimulation During Intrauterine Insemination. American Journal of Clinical and Experimental Medicine. Vol. 4, No. 5, 2016, pp. 134-137. doi: 10.11648/j.ajcem.20160405.14

Received: August 13, 2016; Accepted: September 1, 2016; Published: September 21, 2016

\begin{abstract}
Fecundability increases when ovulation induction was conducted before starting intrauterine insemination procedure (IUI). Human Menopousal gonadotrophin (HMG) and recombinant follicle stimulating hormone (rFSH) are gonadotropin hormones that are widely used in the stimulation of ovulation in infertile patients. Although comparison of gonadotropin preparations have been carried out in IVF, but comparison of rFSH preparations and HMG on IUI was still lacking. The aim of this study was to compare rFSH and HMG, each was combined with clomiphene citrate in infertile women undergone intrauterine insemination (IUI). This study was a retrospective study in the Department of Obstetrics and Gynecology Wahidin Sudirohusodo. Samples were infertile patients who meet these following criteria: women aged 20-45 years, infertility of unknown cause for approximately 12 months, had a history of induced ovulation for approximately 6 cycles without IUI, IVF or ICSI, patent tubes examined with hysterosalpingography, and normal semen analysis according to 1999 WHO criteria. A total of 78 patients consisted of 30 patients using clomiphene citrate $+\mathrm{rFSH}$ and 48 other patients using clomiphene citrate + HMG. In two groups, total dosage, duration of gonadotropin administration, follicle count, mean size of follicles, endometrial line size and pregnancy were compared. Statistical test using Levene $\mathrm{T}$ and unpaired $\mathrm{t}$ test was conducted using SPSS 16 for Windows version. There was no statistically significant difference among total dosage, duration of gonadotropins administration, follicle counts, mean size of follicles, and endometrial line size. The mean number of pregnancy was higher in patients treated with clomiphene citrate $+\mathrm{hMG}$ but this difference was not statistically significant. hMG and $\mathrm{rFSH}$ was equally effective in stimulating ovulation in intrauterine insemination.
\end{abstract}

Keywords: Ovulation Stimulation, Intrauterine Insemination, $\mathrm{HMG}, \mathrm{rFSH}$

\section{Introduction}

Infertility is defined as 1 year of unprotected intercourse without pregnancy. This condition may be further classified as primary infertility, in which no previous pregnancies have occurred, and secondary infertility, in which a prior pregnancy, although not necessarily a live birth, has occurred. The main causes of infertility include male factor, decreased ovarian reserve, ovulatory disorders (ovulatory factor), tubal injury, blockage, or paratubal adhesions (including endometriosis with evidence of tubal or peritoneal adhesions), uterine factors, systemic conditions (including infections or chronic diseases such as autoimmune conditions or chronic renal failure), cervical and immunologic factors, and unexplained factors (including endometriosis with no evidence of tubal or peritoneal adhesions). The basic investigations that should be performed before starting any infertility treatment are semen analysis, confirmation of ovulation, and the documentation of tubal patency. [1] Male factor is the only cause of infertility in $20 \%$ of infertile couples, but it may be a contributing factor in as many as $30 \%$ to $40 \%$ of cases. Treatment of reversible endocrine or infectious causes of subfertility, such as sexually transmitted diseases and thyroid disorders, tends to be effective. 
Intrauterine insemination (IUI) is the best studied and most widely practiced of all the insemination techniques. Intracytoplasmic sperm injection (ICSI) has allowed couples with male factor infertility to achieve assisted reproductive technology (ART) pregnancy outcomes that are comparable with those of couples with non-male factor infertility using conventional in vitro\% fertilization (IVF) treatment.

Ovarian stimulation is being used in the majority of assisted reproduction units in order to improve the success rate by increasing the number of oocytes and thus the number of embryos to be replaced.[2] FSH is the key gonadotropic hormone during the follicular phase and only minute amounts of LH are needed in different stages of follicular development. In IUI, if the cycle is normal, exogenous gonadotropine stimulation can improve the success rate of IUI especially when induction with clomiphene fails or a woman reaches the age of over 35 years.[3] In recent years, induction of ovulation has shown major advances, with multiple products commercially available and the focus of ovarian stimulation has shifted from trying to obtain the maximum possible number of oocytes to trying to obtain an adequate cohort of good quality of embryos from quantity to quality.[4] Urinary products include human menopausal gonadotropins (hMG), urinary FSH (uFSH), and human Chorionic Gonadotropin (hCG). Human menopausal Gonadotropin (HMG) containing $75 \mathrm{IU}$ FSH and $75 \mathrm{IU}$ LH is excreted from the urine of postmenopausal women and is of low purity as $95 \%$ of the proteins are contaminants. The urinary proteins may have negative effects on follicular recruitment and development. [5] More recently, recombinant FSH (r-FSH) and recombinant luteinizing hormone $(\mathrm{rLH})$ have entered the market. Recombinant human FSH (rFSH) is produced by a Chinese hamster ovary cell line, transfected with the genes encoding for the two FSH subunits. The results in an almost totally pure FSH preparation. Finally highly purified (HP)-hMG in which the purification process allows its administration via the subcutaneous route, is the latest addition to this family of infertility drugs. HP-hMG and $\mathrm{rFSH}$ have been widely and successfully used for ovarian stimulation in infertile women undergoing treatment for invitro fertilization/intracytoplasmic sperm injection (IVF/ICSI) and embryo transfer.

It is well known that the quantitative aspects can be modulated by the doses of gonadotropins, the type of gonadotropin used, and by the endocrine environment associated with stimulation. Randomized controlled trials comparing gonadotropin preparations have primarily focused on clinical aspects and have been designed to evaluate the number of oocytes retrieved or, to a lesser extent, pregnancy rates. [6-9]

Several studies comparing the outcome of r-FSH and hMG have been reported, most of which were performed in women undergoing pituitary downregulation with a GnRH agonist long protocol. [10-13] Recent meta analysis have demonstrated that hMG was not inferior to r-FSH with regard to pregnancy and live birth rates. [14-15] Van Wely et al. found a borderline significant difference of a $5 \%$ higher clinical pregnancy rate in women stimulated with menotropins (27\%) compared with r-FSH (22\%). [16] Recently, it was confirmed that a better outcome in terms of the live birth rate was obtained when HP-hMG was used for ovarian stimulation as compared with r-FSH in the GnRH agonist long protocol. [17]

A comparison of gonadotropin preparations have been conducted on IVF, but the comparison of using rFSH preparation and HMG on IUI is still rarely. [18] The aim of this research is to compare the effectiveness between the two preparations gonadotropine (HMG and $\mathrm{rFSH}$ ) that are combined with clomiphene citrate in infertile women using IUI.

\section{Methods}

This research was a retrospective observation in the Obstetrics and Gynecology Department of Wahidin Sudirohusodo Hospital. Samples are infertile patients as many as 78 people who have met the following criteria: female aged between 20-45 years old, unexplained infertility for at least 24 months, history of at least 6 cycles of previous induction ovulation but no IUI, IVF or ICSI attempts, patent fallopian tubes documented by hysterosalpingography (HSG), normal semen analysis according to the 1999 WHO criteria. [19] Patient were excluded if there were history of previous assisted reproduction attempts by IUI, IVF or ICSI, endometriosis (classification stage III and IV of the American Infertility Society), contraindication to one of the investigated drugs, persistent ovarian cyst (larger than $30 \mathrm{~mm}$ and there have been more than 2 months). [18] Samples were divided into two groups. The stimulation of ovulation in Group A was begun with an administration of $50 \mathrm{mg}$ clomiphene citrate daily from the third day until the seventh day of the menstrual cycle, then the patients were given 75 IU of rFSH on the seventh day to ninth day. The method applied in Group B is the same method as group A but the administration of rFSH was replaced with $75 \mathrm{IU}$ hMG. At the tenth day, each sample in both groups are tested on transvaginal ultrasound. All patients were monitored from the tenth day until they reach the dominant follicle $(>17 \mathrm{~mm}$ diameter). Then, they were injected with 5000 IU hCG intramuscularly and the next 24-36 hours IUI procedure was performed. Levene $\mathrm{T}$ and unpaired t-Test was conducted using SPSS 16 for Windows version.

\section{Results}

Table 1. Sample characteristics of this research are valued according to some variables.

\begin{tabular}{llll}
\hline & Group A (rFSH) & Group B (HMG) & $\boldsymbol{p}$ \\
\hline Age & $34.233 \pm 5.210$ & $35.188 \pm 5.8260$ & 0.500 \\
Duration of infertility & $7.00 \pm 3.789$ & $6.25 \pm 4.783$ & 0.266 \\
\hline
\end{tabular}

The table reveals that variables of sample characteristics which are valued have no significant difference between group A (Clomiphene Citrate $+\mathrm{rFSH}$ ) and group $\mathrm{B}$ (Clomiphene Citrate + HMG). 
Table 2. Comparisons of pregnancy and characteristics between group A (Clomiphene Citrate $+r F S H)$ and B (Clomiphene Citrate $+H M G)$.

\begin{tabular}{llll}
\hline & Group A (rFSH) & Group B (HMG) & $p$ \\
\hline Duration of gonadotropin therapy & $5.33 \pm 1.446$ & $5.17 \pm 1.521$ & 0.633 \\
Total doses of gonadotropin & $405.83 \pm 112.319$ & $403.125 \pm 121.4523$ & 0.922 \\
A number of dominant follicles (>17mm) injected with HCG & $1.933 \pm 0.868$ & $2.208 \pm 1.0711$ & 0.240 \\
The average diameter of follicles injected with HCG (mm) & $19.739 \pm 1.268$ & $20.224 \pm 2.208$ & 0.277 \\
Thickness of endometrium injected with HCG (mm) & $7.27 \pm 1.403$ & $7.71 \pm 1.1419$ & 0.181 \\
Total pregnancy & $5(16.66 \%)$ & $7(14.5 \%)$ & 0.522 \\
\hline
\end{tabular}

According to the calculation, total doses of gonadotropin in group A $(\mathrm{rFSH})$ and $\mathrm{B}(\mathrm{HMG})(405.83 \pm 112.319$ vs $403.125 \pm 121.4523 ; \mathrm{p}=0,922)$ and durations of therapy $(5.33 \pm 1.446$ vs $5.17 \pm 1.521 ; \mathrm{p}=0.633)$ have no significant difference. However, in a research of Rashidi et al stated that duration of gonadotropin therapy is shorter in Clomiphene Citrate+ $\mathrm{rFSH}$ group than Clomiphene Citrate +HMG's group.[18] Likewise, with total doses of gonadotropin given to Clomiphene Citrate+ rFSH patients is obtained less than those receiving Clomiphene Citrate+HMG. [17] In this research, both of that doses are insignificant.

In addition, the average of dominant follicles in group A and group $\mathrm{B}$ is insignificant and statistically have no significant difference $(1.933 \pm 0.868$ vs $2.208 \pm 0.9732$; $\mathrm{p}=0.404)$. The average of follicle diameter in group $\mathrm{B}$ is larger than that of group A, however, they statistically have no extremely difference $(19.7390 \pm 1.268$ vs $20.224 \pm 2.208$ $\mathrm{p}=0.277$ ). The thickness of endometrium in group $\mathrm{B}$ is greater than that of group A $(7.27 \pm 1.403$ vs $7.71 \pm 1.1419$; $\mathrm{p}=0.181)$. In group $\mathrm{B}$, there were seven pregnancies $(14.5 \%)$ and group A were five pregnancies $(16.66 \%)$, but this comparisons have no considerable significance $(\mathrm{p}=0.522)$.

\section{Discussions}

Until the recent introduction of $\mathrm{rFSH}$, urinary gonadotropin was used for ovulation induction since the early 1960s and ovarian stimulation since the early 1980s. Besides the difficulty in collecting urine, urinary gonadotropins have a lot of disadvantages including the presence of $\mathrm{LH}$, contamination with $95 \%$ non FSH urinary proteins [5], high incidence of local allergic reactions [20] and batch to batch inconsistency. [21]

Daya et, al in a meta analysis demonstrated that the use of urinary FSH or rFSH was associated with a significantly higher clinical pregnancy rate than HMG. [22] Very low level or the absence of LH in the urinary FSH or $\mathrm{rFSH}$ preparation was considered as the main reason to explain $>50 \%$ improvement in clinical pregnancy rates in women receiving $\mathrm{FSH}$.

Our results are simultaneous with meta analysis of Cochrane that there is no difference in the quantity of follicles, the thickness of endometrium and the average of follicle's size between FSH and HMG, whereas the average of pregnancy in HMG is higher than $\mathrm{rFSH}$. [16] The quantity of pregnancy on patients receiving Clomiphene Citrate + HMG is higher than that of patients receiving Clomiphene Citrate $+\mathrm{rFSH}$ because the level of estradiol is higher after receiving $\mathrm{HMG}$ and it established a good circumstances of endometrium, so that it influences implantation process. [23] Those are reasons why average of pregnancy in group B is higher than that of group A. Another recent study that compared HP-hMG and r-FSH for ovulation induction demonstrated that the LH activity in HP-hMG induces a more modulated folliculogenesis that is associated with a lower risk of excessive ovarian response and an ovulation rate similar to that obtained with r-FSH. [24] In other research concerning about efficacy of lyophilized urinary HMG and rFSH in 49 infertile women who are undergoing IUI is obtained that HP-HMG and rFSH have the same efficacy in stimulation of ovulation. [25] Turhan et al found that HP-HMG to be as effective as $\mathrm{r}$ FSH in terms of oocyte and embryo quality and clinical pregnancy outcomes. [26] From $\mathrm{Ng}$, et al, their study comparing the effects of rFSH and HMG on the quality of oocytes and embryos. They found that $\mathrm{HMG}$ is as good as rFSH in terms of oocyte and embryo quality. [2]

\section{Conclusions}

The use of HMG and rFSH has the same effectiveness in stimulating ovulation in intrautein insemination. A larger population of study is necessary to know the significant effect from using two kinds of the medicines as well as more advanced research to understand the effects of both gonadotropins. Most studies investigated the pregnancy or the delivery rate, but those outcomes are affected with a lot of factors not only quality of embryo, but also number of embryo transferred, local hormone levels, uterine receptivity and other maternal complications. [27]

\section{References}

[1] Berek Jonathan S. Berek \& Novak Gynecology $14^{\text {th }}$ edition, Lippincot, Williams \&Wilkins. 2007.

[2] Ng, E. H, Lau, E. Y. L. Yeung, W. S. B and Ho, P. C. HMG is as good as recombinant human FSH in terms of oocyte and embryo quality: a prospective randomized trial. 2001. Hum, Reprod. 16, 319-325.

[3] Sperrof L, Fritz MA. Clinical gynecologic endocrinology and infertility. $8^{\text {th }}$ ed. Philadelpia: Lippincot Williams and Wilkins; 2011. p. 2337-2341.

[4] Siristatidis C, Trivella M, Chrelias C, Sioulas VD, Vrachnis N, Kassanos D. A short narrative review of the feasibility of adopting mild ovarian stimulation for IVF as the current standard of care. Arch Gynecol Obstet 2012; 286: 505-10. 
[5] Giudice, F. Crisci, C., Eshkol, A, et al. Composition of commercial gonadotropin preparations extracted from human post menopausal urine; characterization of non gonadotropin proteins. Hum. Reprod. 1994; 9, 2291-2299.

[6] Pouwer AW, Farquhar C, Kremer JA. Long acting FSH versus daily FSH for women undergoing assisted reproduction. Cochrane Database Syst Rev 2012; 13; 6CD009577.

[7] Reindolar RH, Goldman MB, Gonadotropin therapy: A $20^{\text {th }}$ century relic. Fertil steril 2012; 97: 813-18.

[8] Beall SA, DeCherney A. History and challenges surrounding ovarian stimulation in the treatment of infertility. Fertil Steril 2012; 97: 795-801.

[9] Loumaye E, Engrand P, Shoham Z, Hillier SG, Baird DT. Clinical evidence of an LH 'ceiling' effect induced by administration of recombinant human LH during the late follicular phase of stimulation cycles in World Health Organization type I and type II anovulation. Hum Reprod 2003; 18: 314-22.

[10] Bjercke S, Tanbo T, Abyholm T, Omland A, Opoien HK, Fedorcsak P. Clinical outcome following stimulation with highly purified $\mathrm{hMG}$ or recombinant FSH in patients undergoing their first treatment cycle of IVF or ICSI. Acta Obstet Gynecol Scand 2010; 89: 1053-60.

[11] Pacchiarotti A, Sbracia M, Frega A, Selman H, Rinaldi L, Pacchiarotti A. Urinary hMG (Meropur) versus recombinant FSH plus recombinant LH (Pergoveris) in IVF: a multicenter, prospective, randomized controlled trial. Fertil Steril 2010; 94: 2467-9.

[12] Ye H, Huang G, Pei L, Zeng P, Luo X. Outcome of in vitro fertilization following stimulation with highly purified $\mathrm{hMG}$ or recombinant FSH in downregulated women of advanced reproductive age: a prospective, randomized and controlled trial. Gynecol Endocrinol 2012; 28: 540-44.

[13] Kilani Z, Dakkak A, Ghunaim S, Cognigni GE, Tabarelli C, Parmegiani L et al. A prospective, randomized, controlled trial comparing HP-hMG with r- FSH in women undergoing ICSI: ovarian response and clinical outcomes. Hum Reprod 2003; 18: 1194-9.

[14] Al-Inany H, Aboulghar M, Mansour R, Serour G. Metaanalysis of recombinant versus urinary derived FSH: an update. Hum Reprod 2003; 18: 305-13.

[15] Al-Inany H, Aboulghra MA, Mansour RT, Seour GI. Ovulation induction in the new millennium: recombinant follicle stimulating hormone versus human menopausal gonadotropin. Gynecol Endocrinol 2005; 20: 161-9.

[16] Van Wely M, Westergaard LG, Bossuyt PM, Van der Veen F. Human Menopousal Gonadotropin versus recombinant Follicle Stimulation hormone for ovarian stimulation in assisted reproductive cycles. Cochrane Database Syst Rev $2011 ; 16$.
[17] Al-Inany HG, Abou-Setta AM, Aboulghar MA, Monsour RT, Serour GI. Efficacy and safety of human menopausal gonadotropins versus recombinant FSH: a meta-analysis. RBM Online 2008; 16: 81-8.

[18] Rashidi, Mandana, et al. Advantages of recombinant FSH over human menopausal gonadatropin for ovarian stimulation in intrauterine insemination: a randomized clinical trial in unexplained infertility. European Journal of Obstetric and Gynecology and Reproductive Biology 2013; 244-247.

[19] World Health Organization. WHO Laboratory manual for the examination of human semen and semen cervical mucus interaction $\left(5^{\text {th }}\right.$ ed). WHO Press, World Health Organization, 1999.

[20] Phipps, W. R, Holden D and Sheehan, R. K. Use of recombinant human follicle stimulating hormone for in vitro fertilization embryo transfer after severe systemic immunoglobulin E-mediated reaction to urofollitropin. 1996. Fertil. steril, 66, 148-150.

[21] Rogers, M., McLoughlin, J. D, Lambert, A. et al. Variability in the immunoreactive and bioactive follicle stimulating hormone content of human urinary menopausal gonadotrophin preparation. Hum. Reprod., 10, 1982-1986.

[22] Daya, S., Gunby, J., Hughes, E. G. et al. Follicle stimulating hormone versus human menopausal gonadotropin for in vitro fertilization cycles: a meta analysis. 1995. Fertil Steril, 64, 347-354.

[23] The European and Israeli Study Group on Highly Menotropin versus Recombinant Follicle Stimulating Hormone. Efficacy and safety of highly purified menotropin versus recombinant follicle stimulating hormone in in vitro fertilization/intracytoplasmic sperm injection cycles: a randomized, comparative trial. Fertil Steril 2002; 78: 520-28.

[24] Platteau P, Andersen AN, Balen A, Devroey P, Sorenson P, Helmgaard L, et al. Similar ovulation rates, but different follicular development with highly purified menotropin compared with r FSH in WHO group II anovulatory infertility: a randomized controlled study. Hum Reprod 2006; 21: 1798804 .

[25] Kocak M, Dilbaz B, Demir B, et al. Lyophilised hMG versus $\mathrm{rFSH}$ in women with unexplained infertility undergoing a controlled ovarian stimulation with intrauterine insemination: a prospective, randomized study. Gynecological Endocrinology 2010; 26: 429-434.

[26] Turhan, N, Pekel A, Ayrim A, Kasap, B, Bayrak, O Effectiveness HP-HMG versus rFSH in patients undergoing IVF/ICSI cycles with moderate male factor infertility. Turk J Med Sci. 2013 43: 144-149.

[27] Tabata, Chisa, Fujiwara, Toshihiro, et. al. Comparison of FSH and $\mathrm{hMG}$ on ovarian stimulation outcome with a GnRH antagonist protocol in younger and advanced reproductive age women.. Reproductive Medicine and Biology; Januari 2015, Vol 14 issue 1, p 5. 